\title{
On the Attainable Order of Runge-Kutta Methods
}

\author{
By J. C. Butcher
}

A Runge-Kutta method with $\nu$ stages for solving the equation

$$
y^{\prime}=f(x, y), \quad y\left(x_{0}\right)=y_{0}
$$

(where $y$ and $f$ are vectors), gives a result

$$
y\left(x_{0}+h\right)=y_{0}+h \sum_{j=1}^{\nu} b_{j} g_{j},
$$

where $g_{1}, g_{2}, \cdots, g_{v}$ satisfy the equations

$$
g_{i}=f\left(x_{0}+c_{i} h, y_{0}+h \sum_{j=1}^{\nu} a_{i j} g_{j}\right), \quad i=1,2, \cdots, \nu .
$$

$a_{11}, a_{12}, \cdots, a_{v v}, b_{1}, b_{2}, \cdots, b_{v}$ are a set of constants which characterize the process and $c_{1}, c_{2}, \cdots, c_{\nu}$ are defined by

$$
c_{i}=\sum_{j=1}^{\nu} a_{i j}, \quad i=1,2, \cdots, \nu .
$$

If the elements of $f$ are differentiable arbitrarily often with respect to $x$ and the elements of $y$, the true solution and the numerical solution can be expanded in powers of $h$. It has been shown [1] that these two expansions agree up to terms in $h^{\rho}$ (that is, the process is of order $\rho$ ) if

$$
\Phi=1 / \gamma \quad \text { whenever } r \leqq \rho .
$$

In this formula, $\Phi$ is a typical elementary weight, $r$ is its order, and $\gamma$ is a certain integral constant associated with it. $\Phi$ itself is a polynomial of degree 1 in $b_{1}, b_{2}, \cdots, b_{\nu}$ and degree $r-1$ in $a_{11}, a_{12}, \cdots, a_{\nu \nu}$.

To find the values of $\gamma$ for the different $\Phi$, one may use a result proved in [1]. If $\Phi_{1}, \Phi_{2}, \cdots, \Phi_{s}$ have values $\gamma_{1}, \gamma_{2}, \cdots, \gamma_{s}$ corresponding to them and if

$$
\Phi=\left[\Phi_{1} \Phi_{2} \cdots \Phi_{s}\right]
$$

then

$$
\gamma=r \gamma_{1} \gamma_{2} \cdots \gamma_{s},
$$

where $r$ is the order of $\Phi$. The notation (2) is used where $\Phi$ is related to $\Phi_{1}, \Phi_{2}, \cdots, \Phi_{s}$ by the formulae

$$
\begin{aligned}
\Phi_{k} & =\sum_{j=1}^{\nu} b_{j} \chi_{k j}, & k=1,2, \cdots, s, \\
\Phi & =\sum_{i=1}^{\nu} b_{i} \prod_{k=1}^{\infty} \sum_{j=1}^{\nu} a_{i j} \chi_{k j}, &
\end{aligned}
$$

and $\chi_{k j}(k=1,2, \cdots, s ; j=1,2, \cdots, \nu)$ is a function of $a_{11}, a_{12}, \cdots, a_{\nu v}$ but not

Received October 27, 1964. 
of $b_{1}, b_{2}, \cdots, b_{p}$. The only elementary weight of order 1 is $\sum_{j=1}^{\nu} b_{j}$ and this will be denoted by $\phi$. For $\phi$ the value of $\gamma$ is 1 .

To illustrate the structure of the various elementary weights consider $\Phi_{1}, \Phi_{2}$, $\Phi_{3}, \Phi$ defined as follows:

$$
\begin{aligned}
\Phi_{1} & =[\phi \phi], \\
\Phi_{2} & =[\phi \phi \phi], \\
\Phi_{3} & =\left[\Phi_{1}\right], \\
\Phi & =\left[\Phi_{1} \Phi_{2} \Phi_{2} \Phi_{3}\right] .
\end{aligned}
$$

These can be simply expressed in terms of the parameters of a process. All summations are from 1 to $\nu$.

$$
\begin{aligned}
\Phi_{1} & =\sum_{i j k} b_{i} a_{i j} a_{i k}=\sum_{i} b_{i} c_{i}^{2}, \\
\Phi_{2} & =\sum_{i} b_{i} c_{i}^{3}, \\
\Phi_{3} & =\sum_{i j} b_{i} a_{i j} c_{j}^{2}, \\
\Phi & =\sum_{i} b_{i}\left(\sum_{j} a_{i j} c_{j}^{2}\right)\left(\sum_{j} a_{i j} c_{j}^{3}\right)^{2}\left(\sum_{j k} a_{i j} a_{j k} c_{k}^{2}\right) .
\end{aligned}
$$

The orders $\left(r_{1}, r_{2}, r_{3}\right.$ and $r$, say) are, respectively, 3, 4, 4, 16 and the constants $\gamma_{1}, \gamma_{2}, \gamma_{3}, \gamma$ are equal to

$$
\begin{aligned}
& \gamma_{1}=3 \cdot 1 \cdot 1=3, \\
& \gamma_{2}=4 \cdot 1 \cdot 1 \cdot 1=4, \\
& \gamma_{3}=4 \cdot 3=12, \\
& \gamma=16 \cdot 3 \cdot 4 \cdot 4 \cdot 12=9216 .
\end{aligned}
$$

It is convenient to write each elementary weight using only brackets and the symbol $\phi$. For the present examples we can write

$$
\begin{aligned}
\Phi_{3} & =\left[\Phi_{1}\right]=[[\phi \phi]], \\
\Phi & =\left[\Phi_{1} \Phi_{2} \Phi_{2} \Phi_{3}\right]=[[\phi \phi][\phi \phi \phi][\phi \phi \phi][[\phi \phi]]] .
\end{aligned}
$$

This notation can be abbreviated by using superscripts to indicate the repetition of blocks of symbols and subscripts to indicate repetition of brackets. With these abbreviations we have

$$
\begin{aligned}
\Phi_{1} & =\left[\phi^{2}\right], \\
\Phi_{2} & =\left[\phi^{3}\right], \\
\Phi_{3} & =\left[{ }_{2} \phi^{2}\right]_{2}, \\
\Phi & =\left[\left[\phi^{2}\right]\left[\phi^{3}\right]^{2}\left[{ }_{2} \phi^{2}\right]_{2}\right] .
\end{aligned}
$$

In this paper we will be concerned only with explicit (that is, classical) processes so that 


$$
a_{i j}=0 \quad \text { whenever } j \geqq i .
$$

With this restriction, there are at least as many parameters $a_{21}, \cdots, b_{\nu}$ to be chosen as there are equations of the form (1), when $\rho=\nu \leqq 4$. It is not surprising, therefore, that there exist Runge-Kutta processes of order $\nu$ with $\nu$ stages when $\nu=1,2$, 3 , 4. However, for $\rho=\nu>4$, there are more equations (1) than there are parameters to choose so it would not be surprising either if there were no Runge-Kutta process of order $\nu$ with $\nu$ stages when $\nu=5,6,7, \cdots$. For the case $\nu=5$, a proof of this result has been given [2]. However, the proof was exceedingly complicated. In this paper the result is proved in a much simpler way for all cases $\nu=5,6,7, \cdots$ and, moreover, it is shown that processes with $\rho+1=\nu$ do not exist when $\rho \geqq 7$. If we use the notation of Antosiewicz and Gautschi [3] and write $p^{*}(\nu)$ for the highest order attainable with $\nu$ stages, then, using results from [2] and this paper, one can determine the following new information about this function:

$$
\begin{aligned}
& p^{*}(5)=4, \\
& p^{*}(6)=5, \\
& p^{*}(7)=6, \\
& p^{*}(8)=6, \\
& p^{*}(\nu) \leqq \nu-2, \quad \nu=9,10, \cdots .
\end{aligned}
$$

The next simplest questions that one might ask are (a) is there a Runge-Kutta process with $\nu=9$ and $\rho=7$; and (b) is there a Runge-Kutta process with $\nu=10$ and $\rho=8$. The first of these must be answered in the affirmative as the author has found such a process; details of this process will be included in a later publication. Thus $p^{*}(9)=7$. The answer to question (b) and, hence, the exact value of $p^{*}(10)$, seems to be still a matter of conjecture.

We will refer to a given process with parameters $a_{21}, a_{31}, \cdots, b_{1}, b_{2}, \cdots$ as the process $P$. Another process with parameters $\bar{a}_{21}, \bar{a}_{31}, \cdots, \bar{b}_{1}, \bar{b}_{2}, \cdots$ will be referred to as $\bar{P}$. $S_{0}(\rho, \nu)$ will denote the set of Runge-Kutta processes of order $\rho$ with $\nu$ stages so that $P \in S_{0}(\rho, \nu)$ if $P$ has $\nu$ stages and the various $\Phi$ formed from the numbers $a_{21}, \cdots, b_{1}, \cdots, b_{\nu}$ satisfy (1). More generally, we write $S_{n}(\rho, \nu)$ for the set of $\nu$ stage processes where, for each $\Phi$ of order $r \leqq \rho,(1)$ is replaced by

$$
\Phi=\frac{r !}{\gamma(n+r) !},
$$

and $n$ is a non-negative integer. Also we write $S(\rho, \nu)$ for the union of the sets $S_{0}(\rho, \nu), S_{1}(\rho, \nu), S_{2}(\rho, \nu), \cdots$.

In terms of this notation we now state the two main results of this paper.

THEOREM 1. The set $S_{0}(\rho, \nu)$ is empty if $\rho \geqq \nu \geqq 5$.

Theorem 2. The set $S_{0}(\rho, \nu)$ is empty if $\rho=\nu-1 \geqq 7$.

A number of lemmas now follow and Theorems 1 and 2 are corollaries to Lemmas 11 and 14.

LEMMA 1. If $U$ and $V$ are $3 \times 3$ matrices with typical elements $u_{i j}$ and $v_{i j}$ and if $U^{T} V$ has only zero elements in the last row and last column but has rank 2 , then either $u_{13}=0$ or $v_{33}=0$. 
Proof. Since $U^{T} V$ is singular, either $U^{T}$ or $V$ is singular. If $U^{T}$ is singular, any (row) null vector of $U^{T}$ is a null vector of $U^{T} V$. Hence, such a vector has only the last component non-zero. Hence the last column of $U$ (and, in particular, $u_{13}$ ) is zero. The other alternative follows similarly.

In applications of Lemma 1 throughout this paper, the matrices $U$ and $V$ will be of the form

$$
\left[\begin{array}{lll}
g_{1}\left(i_{1}\right) & g_{2}\left(i_{1}\right) & g_{3}\left(i_{1}\right) \\
g_{1}\left(i_{2}\right) & g_{2}\left(i_{2}\right) & g_{3}\left(i_{2}\right) \\
g_{1}\left(i_{3}\right) & g_{2}\left(i_{3}\right) & g_{3}\left(i_{3}\right)
\end{array}\right],
$$

where $i_{1}, i_{2}, i_{3}$ are positive integers and $g_{1}(i), g_{2}(i), g_{3}(i)$ are various functions. Such a matrix will be written in the abbreviated form

$$
\left\{g_{1}(i), g_{2}(i), g_{3}(i): i=i_{1}, i_{2}, i_{3}\right\}
$$

and the notation will be extended, where necessary, to matrices of higher order.

At this point it is convenient to introduce also the following notations for $i=1,2, \cdots, \nu$ :

$$
\begin{aligned}
b_{i}{ }^{\prime} & =\sum_{j=1}^{\nu} b_{j} a_{j i}, & c_{i}{ }^{\prime} & =\sum_{j=1}^{\nu} a_{i j} c_{j}, \\
b_{i}{ }^{\prime \prime} & =\sum_{j=1}^{\nu} b_{j}{ }^{\prime} a_{j i}, & c_{i}{ }^{\prime \prime} & =\sum_{j=1}^{\nu} a_{i j} c_{j}^{\prime}, \\
b_{i}^{\prime \prime \prime} & =\sum_{j=1}^{\nu} b_{j}^{\prime \prime} a_{j i}, & c_{i}{ }^{\prime \prime \prime} & =\sum_{j=1}^{\nu} a_{i j} c_{j}^{\prime \prime},
\end{aligned}
$$

so that $b_{\nu}{ }^{\prime}=b_{\nu-1}^{\prime \prime}=b_{\nu}{ }^{\prime \prime}=b_{\nu-2}^{\prime \prime \prime}=b_{\nu-1}^{\prime \prime \prime}=b_{\nu}^{\prime \prime \prime}=c_{2}{ }^{\prime}=c_{2}^{\prime \prime}=c_{3}^{\prime \prime}=c_{2}^{\prime \prime \prime}=c_{3}^{\prime \prime \prime}=c_{4}^{\prime \prime \prime}=0$ as a consequence of $(3)$.

Lemma 2. If $P \in S(\rho, \nu)$ for $\rho, \nu \geqq 2$ then $\bar{P} \in S(\rho-1, \nu-1)$, where $\bar{P}$ is defined by

$$
\begin{aligned}
\bar{a}_{i j} & =a_{i j}, & i, j & =1,2, \cdots, \nu-1, \\
\bar{b}_{j} & =b_{j}{ }_{j}, & j & =1,2, \cdots, \nu-1 .
\end{aligned}
$$

Proof. In fact, we will show that, if $P \in S_{n}(\rho, \nu)$, then $\bar{P} \in S_{n+1}(\rho-1, \nu-1)$. Consider $\Phi=\sum_{j=1}^{\nu} b_{j} \chi_{j}$, an elementary weight for the process $P$ with order $r \leqq \rho-1$. We first prove that $\chi_{j}$ does not depend on any $a_{k l}$ for which $k$ or $l$ exceeds $j$. This is certainly true in the case of $\Phi=\phi$, for, here, $\chi_{j}=1$. The result now follows in other cases by induction on $r$, for, if $\Phi$ is given by (2), then the orders of $\Phi_{1}, \Phi_{2}, \cdots, \Phi_{s}$ are all less than $r$ and

$$
\chi_{j}=\prod_{i=1}^{s} \sum_{k=1}^{\nu} a_{j k} \chi_{i k}
$$

where $\chi_{i k}$, the coefficient of $b_{k}$ in $\Phi_{i}$, by the induction hypothesis, does not depend on any $a_{l m}$ for which $l$ or $m$ exceeds $k$. However, $a_{j k}$ vanishes for $k \geqq j$ so $\chi_{j}$ does not depend on any $a_{l m}$ for which $l$ or $m$ exceeds $j$.

For the process $\bar{P}, \Phi$ takes the value $\sum_{j=1}^{\nu-1} \bar{b}_{j} \chi_{j}$, since $\chi_{j}(j \leqq \nu-1)$ is a function only of the $a_{k l}$ for which $k$ and $l$ are less than $\nu$ and, hence, takes the same value for 
$P$ and $\bar{P}$. We have

$$
\sum_{j=1}^{\nu-1} \bar{b}_{j} \chi_{j}=\sum_{i, j=1}^{\nu} b_{i} a_{i j} \chi_{j}
$$

where we have set the upper limit for $j$ equal to $\nu$, since $a_{i \nu}=0$ for all $i$. This last

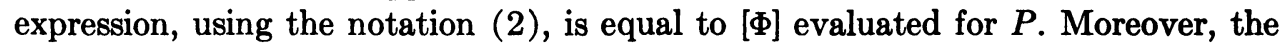
order of $[\Phi]$ is $r+1 \leqq \rho$, so that the constant corresponding to $\gamma$ for this elementary weight is $(r+1) \gamma$. Hence we have

$$
[\Phi]=\frac{(r+1) !}{(r+1) \gamma(r+n+1) !}=\frac{r !}{\gamma(r+n+1) !},
$$

so that $\bar{P} \in S_{n+1}(\rho-1, \nu-1)$.

Lemma 3. If $P \in S_{n}(\rho, \nu)$ for $\rho, \nu \geqq 2$ and $c_{\nu}=1$, then $\bar{P} \in S_{n+1}(\rho-1, \nu-1)$, where $\vec{P}$ is defined by

$$
\begin{aligned}
\bar{a}_{i j} & =a_{i j}, & i, j & =1,2, \cdots, \nu-1, \\
\bar{b}_{j} & =b_{j}\left(1-c_{j}\right) /(n+1), & j & =1,2, \cdots, \nu-1 .
\end{aligned}
$$

Proof. Defining $\Phi, \chi_{j}$ as in the proof of Lemma 2, we have

$$
\sum_{j=1}^{\nu-1} \bar{b}_{j} \chi_{j}=\frac{1}{n+1}\left(\sum_{j=1}^{\nu} b_{j} \chi_{j}-\sum_{j=1}^{\nu} b_{j} c_{j} \chi_{j}\right) .
$$

The first sum on the right-hand side is equal to $\Phi$ evaluated for $P$. If we write $\Phi=\left[\Phi_{1} \Phi_{2} \ldots \Phi_{s}\right]$ and consider the elementary weight $\Phi^{\prime}=\left[\phi \Phi_{1} \Phi_{2} \cdots \Phi_{s}\right]$, we see that the coefficient of $b_{i}$ in $\Phi^{\prime}$ differs from the coefficient of $b_{i}$ in $\Phi$ by the factor

$$
\sum_{j=1}^{n} a_{i j} \cdot 1=c_{i},
$$

since 1 is the coefficient of $b_{i}$ in $\phi$. Hence,

so that

$$
\Phi^{\prime}=\sum_{i=1}^{\nu} b_{i} c_{i} \chi_{i}
$$

$$
\sum_{j=1}^{\nu-1} \vec{b}_{j} \chi_{j}=\frac{1}{n+1}\left(\Phi-\Phi^{\prime}\right)
$$

with the right-hand side evaluated for $P$. Since $P \in S_{n}(\rho, \nu)$, we have

$$
\Phi=\frac{r !}{\gamma(r+n) !} \text {. }
$$

However, the order of $\Phi^{\prime}$ is $r+1 \leqq \rho$ and, corresponding to $\gamma$, it has associated with it the constant $\gamma^{\prime}=(r+1) \cdot 1 \cdot \gamma_{1} \gamma_{2} \cdots \gamma_{s}=(r+1) \gamma / r$, so that

$$
\Phi^{\prime}=\frac{(r+1) !}{((r+1) \gamma / r)(r+n+1) !} .
$$

Subtracting this from the value of $\Phi$ and dividing by $n+1$ we find

$$
\sum_{j=1}^{\nu-1} \bar{b}_{j} \chi_{j}=\frac{r !}{\gamma(r+n+1) !},
$$

so that $\bar{P} \in S_{n+1}(\rho-1, \nu-1)$. 
Lemma 4. If $P \in S(\rho, \nu)$ and $b,=0$, then $\bar{P} \in S(\rho, \nu-1)$, where $\bar{P}$ is defined by

$$
\begin{aligned}
\bar{a}_{i j} & =a_{i j}, & i, j & =1,2, \cdots, \nu-1, \\
\bar{b}_{j} & =b_{j}, & j & =1,2, \cdots, \nu-1 .
\end{aligned}
$$

Lemma 5. If $P \in S(\rho, \nu)$ and $c_{2}=0$, then $\bar{P} \in S(\rho, \nu-1)$, where $\bar{P}$ is defined by

$$
\begin{aligned}
\bar{a}_{i 1} & =a_{i+1,1}+a_{i+1,2}, \\
\bar{a}_{i j} & =a_{i+1, j+1}, \\
\bar{b}_{j} & =b_{j+1}, \\
\bar{b}_{1} & =b_{1}+b_{2} .
\end{aligned}
$$$$
i=1,2, \cdots, \nu-1 \text {, }
$$$$
i, j=2,3, \cdots, \nu-1 \text {, }
$$$$
j=2,3, \cdots, \nu-1 \text {, }
$$

Proofs. These lemmas follow since, for $P$ and $\bar{P}$, any $\Phi$ has the same value. For Lemma 4 this is trivial; for Lemma 5 we consider an elementary weight $\Phi$ which takes the value $\sum_{i=1}^{v} b_{i} \chi_{i}$ for $P$ and $\sum_{i=1}^{\nu-1} \bar{b}_{i} \bar{\chi}_{i}$ for $\bar{P}$. We will prove by induction on $r$, the order of $\Phi$, that

$$
\begin{aligned}
& \chi_{1}=\chi_{2}=\bar{\chi}_{1}, \\
& \chi_{i}=\bar{\chi}_{i-1},
\end{aligned}
$$$$
i=3,4, \cdots, \nu
$$

so that Lemma 5 will follow.

For $\Phi=\phi$ the result is clear. We now suppose $r>1$ and that $\Phi=\left[\Phi_{1} \Phi_{2} \cdots \Phi_{8}\right]$. Since $\Phi_{1}, \Phi_{2}, \cdots, \Phi_{s}$ have orders less than $r$ we may further suppose that

$$
\begin{aligned}
& \chi_{i 1}=\chi_{i 2}=\bar{\chi}_{i 1}, \\
& \chi_{i j}=\bar{\chi}_{i, j-1},
\end{aligned}
$$$$
j=3,4, \cdots, \nu
$$

where we have written, for $i=1,2, \cdots, s$,

$$
\begin{array}{ll}
\Phi_{i}=\sum_{j=1}^{\nu} b_{j} \chi_{i j} & (\text { for } P), \\
\Phi_{i}=\sum_{j=1}^{\nu-1} \bar{b}_{j} \bar{\chi}_{i j} & (\text { for } \bar{P}) .
\end{array}
$$

Since $a_{21}=c_{2}=0$, we now have

$$
\chi_{2}=\prod_{i=1}^{s} \sum_{j=1}^{\nu} a_{2 j} \chi_{i j}=0 .
$$

Also, since $a_{11}=a_{12}=\cdots=\bar{a}_{11}=\bar{a}_{12}=\cdots=0$, we have $\chi_{1}=\bar{\chi}_{1}=0$. Hence, $\chi_{1}=\chi_{2}=\bar{\chi}_{1}$. We also have, for $i=3,4, \cdots, \nu$,

$$
\begin{aligned}
\bar{\chi}_{i-1} & =\prod_{j=1}^{s} \sum_{k=1}^{\nu-1} \bar{a}_{i-1, k} \bar{\chi}_{j k} \\
& =\prod_{j=1}^{\delta}\left(a_{i 1} \chi_{j 1}+a_{i 2} \chi_{j 2}+\sum_{k=2}^{\nu-1} a_{i, k+1} \chi_{j, k+1}\right) \\
& =\prod_{j=1}^{\delta} \sum_{k=1}^{\nu} a_{i k} \chi_{j k} \\
& =\chi_{i} .
\end{aligned}
$$


LEмmA 6. If $c_{\nu-1}=c_{\nu}=1$ and $P \in S(\rho, \nu)$, where $\rho \geqq 2, \nu \geqq 3$, then there is a $\bar{P} \in S(\rho-1, \nu-2)$.

Lemma 7. If $c_{\nu}=1, b_{\nu-1}=0$ and $P \in S(\rho, \nu)$, where $\rho \geqq 2, \nu \geqq 3$, then there is $a$ $\bar{P} \in S(\rho-1, \nu-2)$.

Proofs. To prove Lemmas 6 and 7 we apply Lemma 3 and notice that, in either case, the $\bar{P}$ formed is such that $\bar{b}_{\nu-1}=0$. We now apply Lemma 4 to $\bar{P}$ and the results follow.

Lemma 8. If $\rho>\nu>0$, then $S(\rho, \nu)$ is empty. In particular, $S_{0}(\rho, \nu)$ is empty.

Proof. In view of Lemma 2 and the obvious fact that $S(\rho+1, \nu) \subset S(\rho, \nu)$, it is sufficient to prove that $S(2,1)$ is empty. If $P \in S_{n}(2,1)$, we would have

$$
[\phi]=b_{1} c_{1}=\frac{1}{(2+n) !},
$$

which is not true, since $c_{1}=0$.

Lemma 9. If $P \in S(4,4)$, then $c_{4}=1$.

Proof. If we write

$$
\begin{aligned}
& U=\left\{c_{i}, c_{i}{ }^{2}, 2 c_{i}^{\prime}-c_{i}{ }^{2}: i=2,3,4\right\}, \\
& V=\left\{b_{i}, b_{i} c_{i},(n+1) b_{i}{ }^{\prime}-\left(1-c_{i}\right) b_{i}: i=2,3,4\right\},
\end{aligned}
$$

and suppose that $P \in S_{n}(4,4)$, then the product is

$$
U^{T} V=\left[\begin{array}{ccc}
\frac{1 !}{(n+2) !} & \frac{2 !}{(n+3) !} & 0 \\
\frac{2 !}{(n+3) !} & \frac{3 !}{(n+4) !} & 0 \\
0 & 0 & 0
\end{array}\right] .
$$

The various components in this product are easily verified. For example,

$$
\begin{aligned}
\sum_{i=2}^{4} c_{i}{ }^{2}\left\{(n+1) b_{i}{ }^{\prime}-\left(1-c_{i}\right) b_{i}\right\} & =(n+1)\left[{ }_{2} \phi^{2}\right]_{2}-\left[\phi^{2}\right]+\left[\phi^{3}\right] \\
& =(n+1) \frac{4 !}{12(n+4) !}-\frac{3 !}{3(n+3) !}+\frac{4 !}{4(n+4) !} \\
& =0 .
\end{aligned}
$$

Thus $U, V$ satisfy the conditions of Lemma 1 so that either $2 c_{2}^{\prime}-c_{2}{ }^{2}=0$ or $(n+1) b_{4}{ }^{\prime}-\left(1-c_{4}\right) b_{4}=0$. But $c_{2}{ }^{\prime}=b_{4}{ }^{\prime}=0$ so that either $c_{2}=0$ or $b_{4}=0$ or $c_{4}=1$. The first two alternatives must be rejected as they lead, using Lemmas 5 and 4 , to the existence of a member of $S(4,3)$, which is impossible by Lemma 8 . Hence $c_{4}=1$.

Lemma 10. $S(5,5)$ is empty.

Proof. Suppose $P \in S_{n}(5,5)$. Using the construction of Lemma 2 we can find $\bar{P} \in S(4,4)$ with $\bar{c}_{4}=c_{4}$. Hence $c_{4}=1$. We now use Lemma 1 again with the matrices

$$
\begin{aligned}
U & =\left\{c_{i}, c_{i}{ }^{2}, 2 c_{i}{ }^{\prime}-c_{i}{ }^{2}: i=2,3,5\right\} \\
V & =\left\{b_{i}\left(1-c_{i}\right), b_{i} c_{i}\left(1-c_{i}\right),\left[(n+1) b_{i}{ }^{\prime}-\left(1-c_{i}\right) b_{i}\right]\left(1-c_{i}\right): i=2,3,5\right\}
\end{aligned}
$$


It is easily verified that the product $U^{T} V$ is in the correct form. Note that $i=4$ can be omitted from the various sums defining the elements in $U^{T} V$ since $c_{4}=1$. Hence, we deduce as for the proof of Lemma 9 that $c_{2}=0$ or $b_{5}=0$ or $c_{5}=1$ and, again, the first two alternatives must be rejected. Hence, $c_{5}=c_{4}=1$ so that an application of Lemma 6 yields a member of $S(4,3)$, contrary to Lemma 8 .

LEMMA 11. $S(\rho, \rho)$ is empty if $\rho \geqq \nu \geqq 5$.

Proof. This result follows from Lemmas 2 and 10. Theorem 1 is a corollary. Lemma 12. If $P \in S(6,7)$, then $c_{7}=1$.

Proof. We suppose $P \in S_{n}(6,7)$. Consider the matrices

$$
\begin{array}{r}
U=\left\{c_{i}, c_{i}{ }^{2}, c_{i}{ }^{3}, 2 c_{i}{ }^{\prime}-c_{i}{ }^{2},\left(2 c_{i}{ }^{\prime}-c_{i}{ }^{2}\right)\left(c_{i}-c_{2}\right), 3 c_{i}{ }^{\prime \prime}-c_{i}{ }^{\prime} c_{i}: i=2,3,4,5,6,7\right\}, \\
V=\left\{b_{i}, b_{i} c_{i}, b_{i} c_{i}{ }^{2},(n+1) b_{i}{ }^{\prime}-b_{i}\left(1-c_{i}\right),\left[(n+1) b_{i}{ }^{\prime}-b_{i}\left(1-c_{i}\right)\right]\left(c_{i}-c_{7}\right),\right. \\
\left.(n+2) b_{i}{ }^{\prime \prime}-b_{i}{ }^{\prime}\left(1-c_{i}\right): i=2,3,4,5,6,7\right\} .
\end{array}
$$

It is found that

$$
U^{T} V=\left[\begin{array}{ll}
W & O \\
O & O
\end{array}\right]
$$

where $W$ is a nonsingular $3 \times 3$ matrix and $O$ denotes a zero matrix. Hence, either $U$ or $V$ has rank less than 5. Suppose it is $U$. Since a (row) null vector of $U^{T}$ is also a null vector of $U^{T} V$, such a vector must have the first three components zero. If there were a null vector with the fourth component non-zero, it would follow that $2 c_{2}^{\prime}-c_{2}^{2}=0$ since $\left(2 c_{i}{ }^{\prime}-c_{i}{ }^{2}\right)\left(c_{i}-c_{2}\right)$ and $3 c_{i}^{\prime \prime}-c_{i}{ }^{\prime} c_{i}$ both vanish when $i=2$. If there were no such null vector, it would follow that (000010) and (000001) are each null vectors so that $\left(2 c_{3}{ }^{\prime}-c_{3}{ }^{2}\right)\left(c_{3}-c_{2}\right)=3 c_{3}{ }^{\prime \prime}-c_{3}{ }^{\prime} c_{3}=0$. In the case when $V$ has rank less than 5 it would follow similarly that $(n+1) b_{7}{ }^{\prime}-$ $b_{7}\left(1-c_{7}\right)=0$ or $\left[(n+1) b_{6}{ }^{\prime}-b_{6}\left(1-c_{6}\right)\right]\left(c_{6}-c_{7}\right)=(n+2) b_{6}{ }^{\prime \prime}-b_{6}{ }^{\prime}\left(1-c_{6}\right)=0$. Using the fact that $c_{2}^{\prime}=c_{3}{ }^{\prime \prime}=b_{7}{ }^{\prime}=b_{6}^{\prime \prime}=0$, the four alternatives simplify to

$$
\begin{aligned}
c_{2} & =0, \\
\left(2 c_{3}{ }^{\prime}-c_{3}{ }^{2}\right)\left(c_{3}-c_{2}\right) & =c_{3}{ }^{\prime} c_{3}=0, \\
b_{7}\left(1-c_{7}\right) & =0, \\
{\left[(n+1) b_{6}{ }^{\prime}-b_{6}\left(1-c_{6}\right)\right]\left(c_{6}-c_{7}\right) } & =b_{6}{ }^{\prime}\left(1-c_{6}\right)=0 .
\end{aligned}
$$

The possibility that $c_{2}=0$ is rejected as it would imply the existence of a member of $S(6,6)$. Assuming that $c_{2} \neq 0,(5)$ implies that $c_{3}{ }^{\prime}=0$. We now use Lemma 1 with

$$
\begin{aligned}
& U=\left\{c_{i}^{\prime}, c_{i}^{\prime} c_{i}, 3 c_{i}^{\prime \prime}-c_{i}^{\prime} c_{i}: i=4,5,6\right\}, \\
& V=\left\{b_{i}^{\prime}, b_{i}^{\prime} c_{i},(n+2) b_{i}^{\prime \prime}-b_{i}^{\prime}\left(1-c_{i}\right): i=4,5,6\right\},
\end{aligned}
$$

and deduce that $c_{4}{ }^{\prime} c_{4}=0$ or $b_{6}{ }^{\prime}\left(1-c_{6}\right)=0$. If $c_{4}{ }^{\prime}=0$, we have $0=\sum_{i=1}^{7} b_{i}{ }^{\prime \prime \prime} c_{i}{ }^{\prime}=$ $1 /(n+6)$ !, a contradiction. If $c_{4}=0$, we use Lemma 1 with

$$
\begin{aligned}
& U=\left\{c_{i}^{\prime} c_{i}, c_{i}^{\prime} c_{i}{ }^{2}, 4 c_{i}^{\prime \prime \prime}-c_{i}^{\prime \prime} c_{i}: i=5,6,7\right\}, \\
& V=\left\{b_{i}, b_{i} c_{i},(n+1) b_{i}{ }^{\prime}-b_{i}\left(1-c_{i}\right): i=5,6,7\right\},
\end{aligned}
$$

to deduce that $c_{5} c_{5}{ }^{\prime \prime}=0$ or $b_{7}\left(1-c_{7}\right)=0$. If $c_{5}^{\prime \prime}=0$ (and also $c_{4}^{\prime \prime}=0$, since 
$\left.c_{3}^{\prime}=0\right)$ we find $0=\sum_{i=1}^{7} b_{i}{ }^{\prime \prime} c_{i}^{\prime \prime}=1 /(n+6) !$, a contradiction. If $c_{5}=0$, we find $0=\sum_{i=1}^{7} b_{i}^{\prime \prime} c_{i} c_{i}^{\prime}=3 /(n+6) !$, a contradiction. The possibility $b_{7}=0$ is immediately rejected as it leads, by Lemma 4 , to the existence of a member of $S(6,6)$. Thus the present alternative leads to the result $c_{7}=1$.

Still assuming $c_{3}{ }^{\prime}=0$, we now consider the possibility that $b_{6}{ }^{\prime}\left(1-c_{6}\right)=0$. We must reject the possibility that $b_{6}{ }^{\prime}=0$, since $S(5,5)$ is empty. Hence $c_{6}=1$. We now apply Lemma 1 once more with

$$
\begin{aligned}
& U=\left\{c_{i}^{\prime}, c_{i}^{\prime} c_{i}, 3 c_{i}^{\prime \prime}-c_{i}^{\prime} c_{i}: i=4,5,7\right\}, \\
& V=\left\{b_{i}\left(1-c_{i}\right), b_{i} c_{i}\left(1-c_{i}\right),\left[(n+1) b_{i}^{\prime}-b_{i}\left(1-c_{i}\right)\right]\left(1-c_{i}\right): i=4,5,7\right\},
\end{aligned}
$$

and deduce that $c_{4}^{\prime} c_{4}=0$ (previously rejectęd) or $b_{7}\left(1-c_{7}\right)=0$. Again this leads to the result that $c_{7}=1$.

We now pass on to the alternatives (6) and (7). (6) implies that $c_{7}=1$. If $c_{7} \neq 1,(7)$ implies $b_{6}{ }^{\prime}=0$ which leads to a contradiction to Lemma 10.

Lemma 13. $S(7,8)$ is empty.

Proof. We suppose there is a $P \in S_{n}(7,8)$. Using the construction of Lemma 2, we see that Lemma 12 may be used to deduce that $c_{7}=1$. We recall also, from the proof of Lemma 12, that either $c_{8}^{\prime}=c_{4}=0$, or $c_{0}=1$ or neither of $(4),(5)$ is satisfied.

If $c_{3}{ }^{\prime}=c_{4}=0$, we use Lemma 1 with

$U=\left\{c_{i}^{\prime} c_{i}, c_{i}^{\prime} c_{i}^{2}, 4 c_{i}^{\prime \prime \prime}-c_{i}^{\prime \prime} c_{i}: i=5,6,8\right\}$,

$V=\left\{b_{i}\left(1-c_{i}\right), b_{i} c_{i}\left(1-c_{i}\right),\left[(n+1) b_{i}{ }^{\prime}-b_{i}\left(1-c_{i}\right)\right]\left(1-c_{i}\right): i=5,6,8\right\}$,

to deduce that $c_{5} c_{5}^{\prime \prime}=0$ (rejected as in the proof of Lemma 12) or else $b_{8}\left(1-c_{8}\right)^{2}$ $=0$. Hence $c_{8}=1$ and we use Lemma 6 to find a member of $S(6,6)$, contrary to Lemma 11.

If $c_{6}=c_{7}=1$ we again use Lemma 6 to find a member of $S(5,5)$, which is impossible by Lemma 10. Hence, neither (4) nor (5) is satisfied. We now use the type of argument used at the start of the proof of Lemma 12 with the matrices

$$
\begin{array}{r}
U=\left\{c_{i}, c_{i}{ }^{2}, c_{i}{ }^{3}, 2 c_{i}{ }^{\prime}-c_{i}{ }^{2},\left(2 c_{i}{ }^{\prime}-c_{i}{ }^{2}\right)\left(c_{i}-c_{2}\right), 3 c_{i}{ }^{\prime \prime}-c_{i}{ }^{\prime} c_{i}:\right. \\
i=2,3,4,5,6,8\}, \\
V=\left\{b_{i}\left(1-c_{i}\right), b_{i} c_{i}\left(1-c_{i}\right), b_{i} c_{i}{ }^{2}\left(1-c_{i}\right),\left[(n+1) b_{i}{ }^{\prime}-b_{i}\left(1-c_{i}\right)\right]\left(1-c_{i}\right),\right. \\
\left.(n+3) \sum_{j=1}^{8} b_{j}{ }^{\prime}\left(1-c_{j}\right) a_{j i}-b_{i}{ }^{\prime}\left(1-c_{i}\right)^{2}: i=2,3,4,5,6,8\right\},
\end{array}
$$

to deduce, since the rank of $U$ exceeds 4 , that one of the following results holds:

$$
\begin{aligned}
{\left[(n+1) b_{8}{ }^{\prime}-b_{8}\left(1-c_{8}\right)\right]\left(1-c_{8}\right) } & =0, \\
(n+3) \sum_{j=1}^{8} b_{j}{ }^{\prime}\left(1-c_{j}\right) a_{j 6}-b_{6}{ }^{\prime}\left(1-c_{6}\right)^{2} & =0,
\end{aligned}
$$

and this implies that $b_{6}{ }^{\prime}$ or $b_{8}$ is zero or that $c_{6}$ or $c_{8}$ is unity. If $b_{6}{ }^{\prime}=0$ we use Lemma 2 followed by Lemma 7 to obtain a member of $S(5,5)$, contrary to Lemma 10 . The remaining possibilities have all been previously considered and disposed of. 
LEMMA 14. $S(\rho, \nu)$ is empty for $\rho+1 \geqq \nu \geqq 8$.

Proof. The result follows from Lemma 2 and Lemma 13. Theorem 2 is a corollary.

University of Canterbury

Christchurch, New Zealand

Present address:

Stanford Linear Accelerator Center

Stanford, California

1. J. C. Butcher, "Coefficients for the study of Runge-Kutta integration processes," J. Austral. Math. Soc., v. 3, 1963, pp. 185-201. MR 27 *2109.

2. J. C. Butcher, "On Runge-Kutta processes of high order," J. Austral. Math. Soc., v. 4, 1964, pp. 179-194. MR $29 * 2972$.

3. H. A. ANTOSIEWICZ \& W. GAUTSCHI, "Numerical methods in ordinary differential equations," A Survey of Numerical Analysis, McGraw-Hill, New York, 1962, pp. 314-346. MR 24 * B2114. 